\title{
Microvesicles released from human embryonic stem cell derived-mesenchymal stem cells inhibit proliferation of leukemia cells
}

\author{
YUAN JI $^{1}$, YONGBIN MA ${ }^{1}$, XIANG $\mathrm{CHEN}^{1}$, XIANYAN JI ${ }^{1}$, JIANYI GAO ${ }^{1}$, LEI ZHANG ${ }^{1}$, KAI YE ${ }^{1}$,

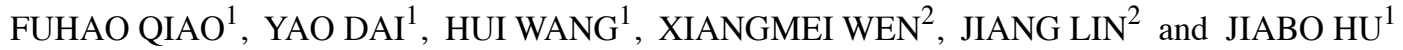 \\ ${ }^{1}$ School of Medicine, Jiangsu University, Zhenjiang, Jiangsu 212013; ${ }^{2}$ Laboratory Center, \\ Affiliated People's Hospital of Jiangsu University, Zhenjiang, Jiangsu 212002, P.R. China
}

Received December 29, 2016; Accepted May 29, 2017

DOI: $10.3892 /$ or.2017.5729

\begin{abstract}
Human embryonic stem cell derived-mesenchymal stem cells (hESC-MSCs) are able to inhibit proliferation of leukemia cells. Microvesicles released from human embryonic stem cell derived-mesenchymal stem cells (hESC-MSC-MVs) might play an important part in antitumor activity. Microvesicles were isolated by ultracentrifugation and identified under a scanning electron microscopy and transmission electron microscope separately. After 48-h cocultured with hESC-MSCs and hESC-MSC-MVs, the number of K562 and HL60 was counted and tumor cell viability was measured by CCK8 assay. The expression of proteins Bcl-2 and Bax were estimated by western blotting. Transmission electron microscope and western blot analysis were adopted to evaluate the autophagy level. Results showed that both hESC-MSCs and hESC-MSC-MVs inhibited proliferation of leukemia cells in a concentration-dependent manner. hESC-MSC-MVs reduced the ratio of $\mathrm{Bcl} / \mathrm{Bax}$, enhanced the protein level of Beclin-1 and LC3-II conversion, thus upregulating autophagy and apoptosis. In conclusion, microvesicles released from human embryonic stem cell derived-mesenchymal stem cells inhibited tumor growth and stimulated autophagy and excessive autophagy might induce apoptosis.
\end{abstract}

\section{Introduction}

Mesenchymal stem cells (MSCs) are a group of cells with the capacity of self-renewal and multilineage differentiation. Recent studies showed that MSCs could be used as cellular vectors for targeted delivery of therapeutic agents to tumors (1). MSCs can be derived from a variety

Correspondence to: Dr Jiabo Hu, School of Medicine, Jiangsu University, 301 Xuefu Road, Zhenjiang, Jiangsu 212013, P.R. China

E-mail: hu@ujs.edu.cn

Key words: microvesicles, human embryonic stem cell derivedmesenchymal stem cells, leukemia, apoptosis, autophagy of tissue sources such as bone marrow, umbilical cord and adipose tissue, however, these adult stem cell sources were still inadequate for clinical use due to their limited supply and expansion, and further more, inevitable invasion when collected (2). Fortunately, a new source of mesenchymal stem cells emerged at a historic moment - human embryonic stem cell-derived mesenchymal cells (hESC-MSCs). hESC-MSCs are easier to be standardized, non-invasive and greatly proliferate in vitro, which might be an alternative for traditional adult stem cell therapy (3).

Microvesicles (MVs) are small, spherical membrane fragments, 50-1,000 $\mathrm{nm}$ in diameter and believed to be involved in multiple aspects of cancer progression $(4,5)$. It was reported that MVs could deliver antitumor microRNAs, thus inhibiting tumor growth and stimulating apoptosis (6). Recent studies demonstrated that human umbilical cord blood-derived mesenchymal stem cells (UC-MSCs) inhibited the growth of K562 (7). Particularly, stem cells are a rich source of soluble factors and MVs. Based on these findings, we assumed that hESC-MSCs might also suppress the growth of leukemia cells, and in which MVs probably played an important role.

\section{Materials and methods}

Cell culture. The human embryonic stem cell line (NJ15) was kindly provided by Dr Lianju Qin, from the Nanjing Maternity and Child Health Care Hospital, Nanjing, China under a Materials Transfer Agreement. Human umbilical cord-derived mesenchymal stem cell (UC-MSCs) and bone marrow-derived mesenchymal stem cells (BM-MSCs) were provided by Dr Wei Zhu, School of Medicine, Jiangsu University. K562 and HL60 cells were provided by Dr Jun Qian, Affiliated People's Hospital of Jiangsu University. The hESCs were cultured on mitomycin C (Roche, USA)-pretreated mouse embryonic fibroblast (MEF) and maintained in the hESCs medium: DMEM/F12 (Gibco, Grand Island, NY, USA) $80 \mathrm{ml}$, knockout serum replacement (Gibco) $20 \mathrm{ml}, 1 \%$ non-essential amino acid (Gibco), $1 \mathrm{mM}$ L-glutamine (Gibco), $0.1 \mathrm{mM}$ $\beta$-mercaptoethanol (Sigma Chemical Co., St. Louis, MO, USA), $4 \mathrm{ng} / \mathrm{ml}$ basic fibroblast growth factor (Gibco). 
We harvested hESC-MSCs based our previous study (8). Briefly, hESCs were directly seeded onto a plate coated with $0.1 \%$ gelatin and cultured in the MSC growth medium: DMEM low-glucose medium supplemented with $10 \%$ fetal bovine serum (ExCell Bio, FND500, Australia). One week later, cells were passaged and cultured in normal subculture (Named P1). After serial passages, further purification was achieved.

Human UC-MSCs, BM-MSCs and leukemia cell lines K562 and HL60 were maintained in DMEM medium (Gibco) supplemented with 10\% FBS.

Cell growth and cell viability assays. To evaluate the effects of hESC-MSCs on the proliferation of K562 and HL60, $1 \times 10^{5}$ leukemia cells/well were cultured solely (as control) or incubated with hESC-MSCs in DMEM medium without serum (hESC-MSCs were pre-treated by mitomycin $\mathrm{C}$ and cultured overnight prior to coculture with leukemia cells). After 48-h coculture, leukemia cells were collected and counted under a microscope. For Transwell cocultures (Transwell, 3- $\mu \mathrm{m}$ pore size; Corning, USA), hESC-MSCs were plated at a concentration of $1 \times 10^{5}$ cells/well in the upper compartment and an equal number of K562 and HL60 cells were plated in the lower compartment $24 \mathrm{~h}$ later. In direct contact groups, $1 \times 10^{5}$ leukemia cells were in coculture with hESC-MSCs at ratios of 50:1 and 0.5:1 of hESC-MSCs. After 24-h coculture, leukemia cells were collected and assessed with a Cell Counting Kit-8 (CCK-8) assay. To evaluate the effects of MVs on the growth of leukemia cells, K562 and HL60 were incubated with different dose of MVs in DMEM medium without serum. Forty-eight hours later, cell viability was analyzed by CCK- 8 method. Into each well, $100 \mu 1$ cell suspension was added with $10 \mu$ l CCK-8 reagent (Dojindo, Japan) and incubated for $2 \mathrm{~h}$ at $37^{\circ} \mathrm{C}$. Finally, the optical density (OD) values were read on an enzyme-labeled ELx800 (Bio-Tek, Winooski, VT, USA) at $450 \mathrm{~nm}$. We calculated the proliferation inhibition rate (IR) by the following formula: IR = [1 - (mean OD value of test groups) / (mean OD value of control groups)] x $100 \%$.

CCK-8 assay was used to determine the number of living cells in cell proliferation assays by utilizing [2-(2-methoxy4-nitrophenyl)-3-(4-nitrophenyl)-5-(2, 4-disulfophenyl)2H-tetrazolium, monosodium salt] (WST-8). WST-8 decreased by dehydrogenases in cells to give an orange colored product (formazan), which was directly proportional to the number of viable cells.

$M V$ s isolation and characterization by electron microscopy. To collect MVs, hESC-MSCs were cultured in DMEM medium depleted of serum. Forty-eight hours later hESC-MSC-MVs were purified following the standardized procedure reported by Gadkari et al and Bruno et al $(9,10)$. In brief, the supernatants (or conditioned medium) of hESC-MSCs were centrifuged at $2,000 \mathrm{x} \mathrm{g}$ for $5 \mathrm{~min}$ and then $12,000 \mathrm{x} \mathrm{g}$ for $15 \mathrm{~min}$ to remove cell fragments and impurities. Then, conditioned medium was centrifuged (Beckman Coulter Optima L-90K, Beckman Coulter, Fullerton, CA, USA) at 100,000 x g for at least $1 \mathrm{~h}$, washed in the DMEM containing $25 \mathrm{mM}$ HEPES (Gibco) and followed by a second ultracentrifugation in the same way. We removed the excess fluid and added $200 \mu \mathrm{l}$ PBS to resuspend the pellets. The protein content of hESC-MSC-MVs was analyzed by BCA method (Sigma).
To ensure that the isolated pellets were hESC-MSC-MVs, we identified them under a scanning electron microscope (SEM; Hitachi, Japan) and a transmission electron microscope (TEM; Philips, FEI, Eindhoven, The Netherlands) respectively.

Characterization of autophagosomes by TEM. Leukemia cells were first fixed by $3 \%$ glutaraldehyde plus $2 \%$ formaldehyde in $0.1 \mathrm{M}$ sodium cacodylate buffer solution. Then cells were fixed with $2 \%$ osmium tetroxide and dehydrated with propylene oxide and ethanol. After that, cells were embedded, and ultrathin sections were cut and stained in uranyl acetate as well as lead citrate, and observed under a TEM (Philips).

Western blot analysis. Leukemia cells $\left(1 \times 10^{5}\right.$ cells/well $)$ were cultured solely (as control) or incubated with hESC-MSC-MVs (30 and $60 \mu \mathrm{g} / \mathrm{ml}$ ) in DMEM medium without serum. Forty-eight hours later, total protein of leukemia cells was extracted and $100 \mu \mathrm{g}$ protein was subjected to $12 \%$ SDS-PAGE and subsequently electroblotted onto PVDF membranes. These reactive bands were analyzed with enhanced chemiluminescence (ECL, Millipore, USA) reagents. Signals were captured using an Image Quant ${ }^{\mathrm{TM}}$ LAS-4000 Mini Imager (GE Healthcare, Piscataway, NJ, USA). All primary antibodies including rabbit anti-GAPDH (cat. no. 5174), anti-Beclin-1 (cat. no. 3738), anti-LC3B (cat. no. 2775), anti-Bax (cat. no.2772), anti-Bcl-2 (cat. no. 2876) were purchased from Cell Signaling Technology (CST Beverly, MA, USA). Primary antibodies were detected using goat anti-rabbit secondary antibody (Thermo Fisher Scientific, Rockford, IL, USA). The protein density of each band was detected using Quantity One v4.62 software (Bio-Rad, Inc, Berkeley, CA, USA) for semiquantitative evaluation.

Flow cytometer analysis. Apoptosis of tumor cells was measured using Annexin V-FITC/PI apoptosis kit (BD Biosciences, San Jose, CA, USA). In total, $1 \times 10^{5}$ leukemia cells were collected and stained with $5 \mu \mathrm{l}$ Annexin V-FITC and PI successively under normal temperature for 15 min away from light, and analyzed within an hour on flow cytometer (FACSCalibur; BD Biosciences).

Statistical analysis. The one-way ANOVA with multiple comparisons was adopted to evaluate the difference among three or more groups. Each experiment was independently carried out at least three times. Data are shown as the mean \pm standard deviation (SD) and p-value $\leq 0.05$ was statistically significant.

\section{Results}

Generation of human embryonic stem cell derivedmesenchymal stem cells (hESC-MSCs). hESCs (P31) were originally cultured under feeder-dependent conditions (Fig. 1A). Approximately 3 days after cultured in MSC medium, long spindle cells began appearing around the colonies. When these long spindle cells reached $>75 \%$ confluence ( $\sim 7$ days later), cells were digested and seeded onto new gelatin-coated cultures. With the removal of colony structures, heterogeneous cells became homogeneous and gradually began to be more slender and confluent (Fig. 1B and C). At passage 10, some shuttle fibroblast-like cells were 


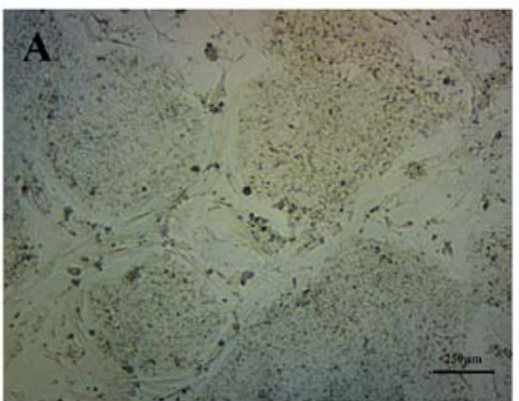

hESCs P31

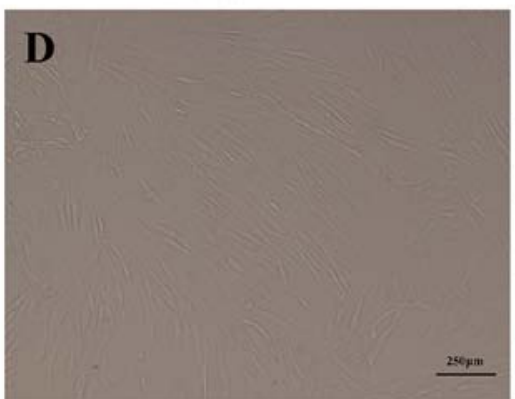

hESC-MSCs P10

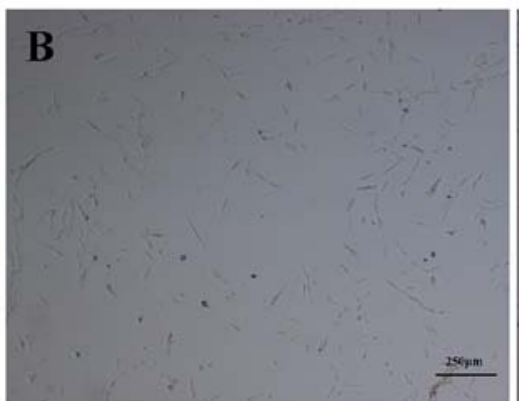

hESC-MSCs P2

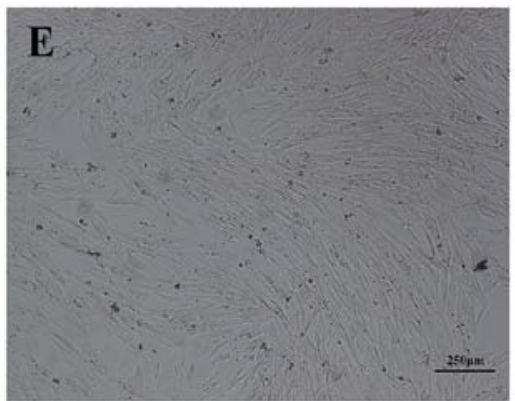

UC-MSCs P3

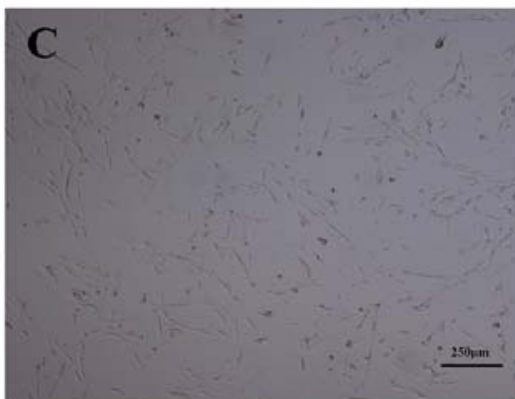

hESC-MSCs P5

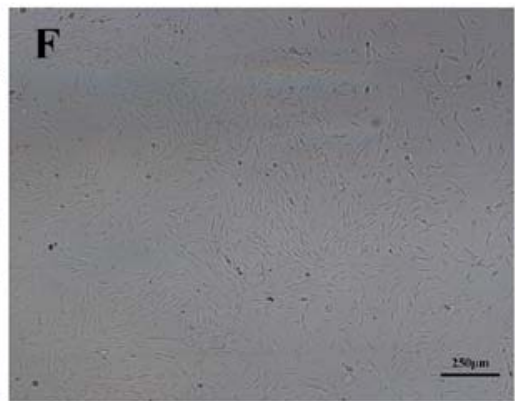

BM-MSCs P3

Figure 1. Morphology of human embryonic stem cell derived-mesenchymal stem cells. (A) Human embryonic stem cells were cultured on mouse embryonic fibroblasts. (B and C) Fibroblast-like cells began to move out of the cell clones after cultured in the mesenchymal stem cells in growth medium for a week. With increased passages, cells tended to be more slender and arrange more orderly. (D) At passage 10, some shuttle fibroblast-like cells were in the majority and arranged regularly similar to (E) umbilical cord-derived mesenchymal stem cells and (F) bone marrow-derived mesenchymal stem cells. Scale bars, $250 \mu \mathrm{m}$.
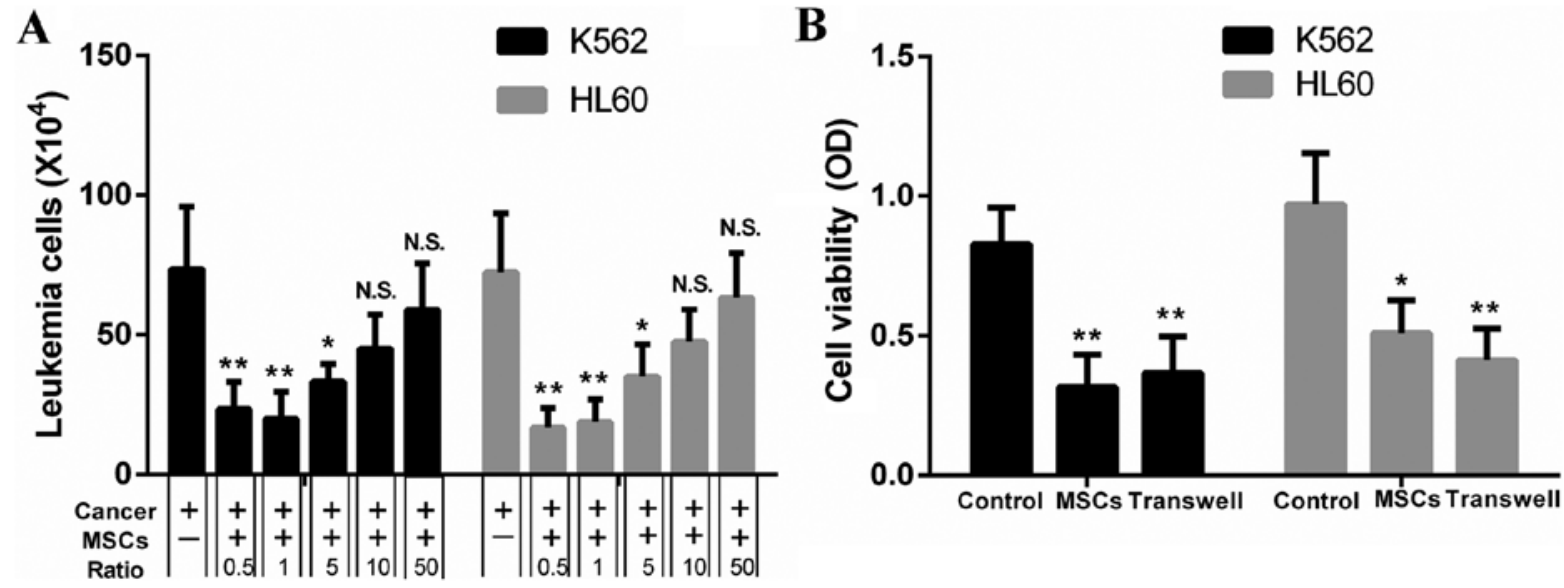

Figure 2. Human embryonic stem cell derived-mesenchymal stem cells inhibit proliferation of leukemia cells. (A) After 48-h coculture, human embryonic stem cell derived-mesenchymal stem cells showed an inhibitory effect on proliferation of leukemia cells, furthermore, this effect decreased with the increasing ratio of leukemia cells to human embryonic stem cell derived-mesenchymal stem cells. (B) Similarly, this inhibitory effect still existed in Transwell cocultures. $\mathrm{n}=3$, ${ }^{*} \mathrm{p}<0.05,{ }^{* *} \mathrm{p}<0.01$. NS, not significant, all treatment groups were compared with a control group.

in the majority and arranged regularly similar to UC-MSCs and BM-MSCs (Fig. 1D-F).

hESC-MSCs inhibit the proliferation of leukemia cells. We observed that hESC-MSCs showed an inhibitory effect on proliferation of leukemia cells which was concentrationdependent. The number of K562 and HL60 cells alone, cocultured with hESC-MSCs at ratios of 50:1 and 0.5:1 as shown in Fig. 2, the amount of K562 and HL60 cells rose with the increasing ratio of leukemia cells to hESC-MSCs over the range of $0.5: 1-50: 1$ (Fig. 2A). To determine whether this effect was realized through paracrine or cell-to-cell contact, the Transwell chamber technology was used and CCK8-assay was adopted to evaluate cell viability. The inhibition rate of K562 for direct contact and Transwell coculture groups was $61.3 \pm 15.7$ versus $51.3 \pm 4.6 \%$. In HL60, the rate was $46.6 \pm 13.2$ versus $57.2 \pm 8.8 \%$ and there was no significant difference between these groups. Results indicated that cell-to-cell contact might not be necessary, since this effect still existed in Transwell cocultures (Fig. 2B). 


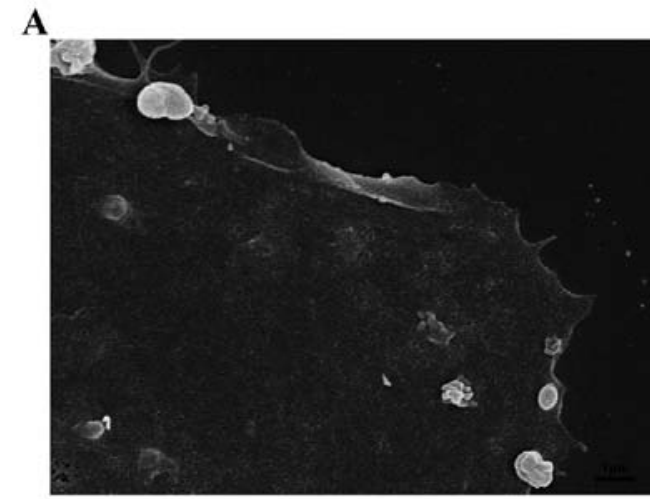

SEM

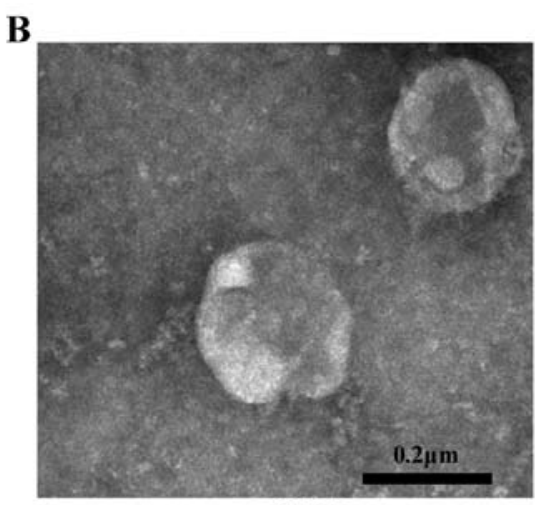

TEM

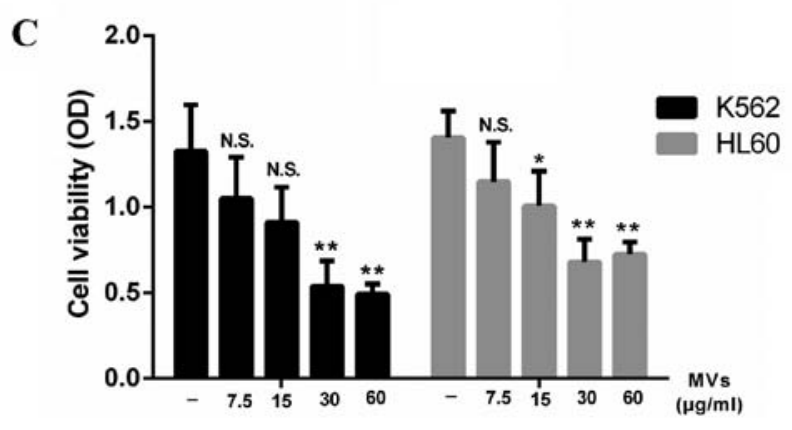

Figure 3. Microvesicles released from human embryonic stem cell derived-mesenchymal stem cells inhibit proliferation of leukemia cells. Through ultracentrifugation, microvesicles were isolated from supernatants of human embryonic stem cell derived-mesenchymal stem cells cultured in serum-free medium for $48 \mathrm{~h}$. (A) Scanning electron microscope figure shows the secretion process of MVs which were being released from the cell membrane surface. Scale bars, $1 \mu \mathrm{m}$. (B) Under transmission electron microscope, microvesicles were small, spherical membrane fragments. Scale bars, $0.2 \mu \mathrm{m}$. (C) The result of CCK- 8 assay indicated that microvesicles inhibited the growth of leukemia cells in a concentration-dependent manner. $\mathrm{n}=3,{ }^{*} \mathrm{p}<0.05$, ${ }^{* *} \mathrm{p}<0.01$. NS, not significant, all treatment groups were compared with a control group.

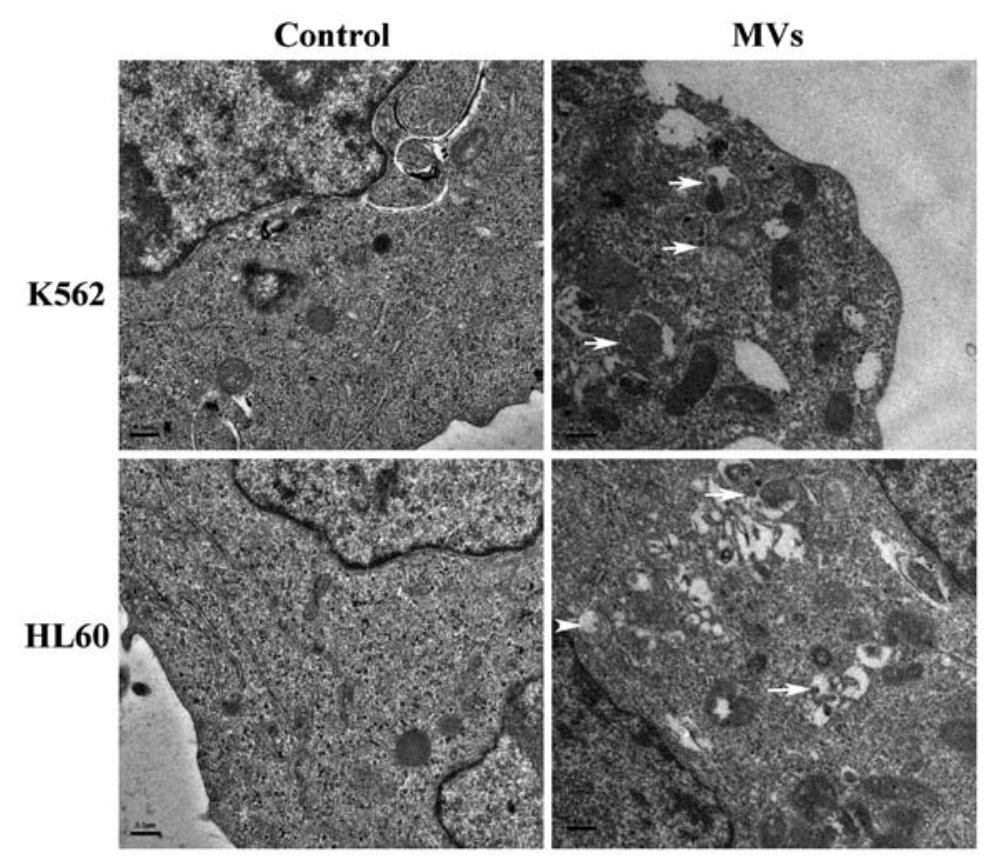

Figure 4. Under a transmission electron microscope, autophagosomes are seen in leukemia cells. In experimental groups, K562 cells and HL60 cells incubated with microvesicles were found with many electron-dense vacuoles, which contained extensively degraded organelles (white arrow). Scale bars, $0.3 \mu \mathrm{m}$.

hESC-MSCs-MVs inhibit the proliferation of leukemia cells. Supernatants of hESC-MSCs were ultracentrifuged and the collected pellets were captured under a SEM and TEM separately. We observed that the hESC-MSC-MVs were small, spherical membrane fragments, several hundreds nanometers in diameter (Fig. 3B) and released from cell surface or by 
A

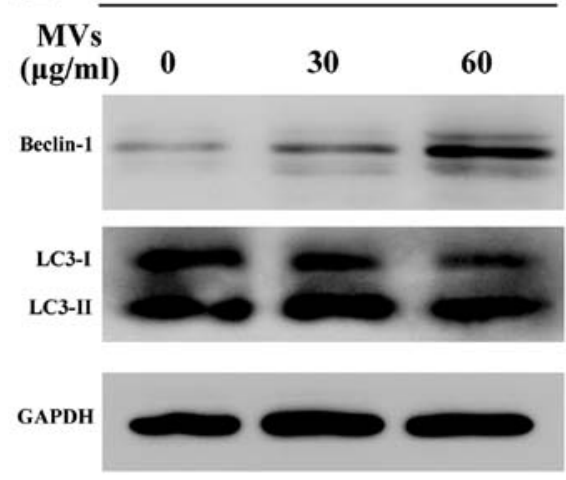

B
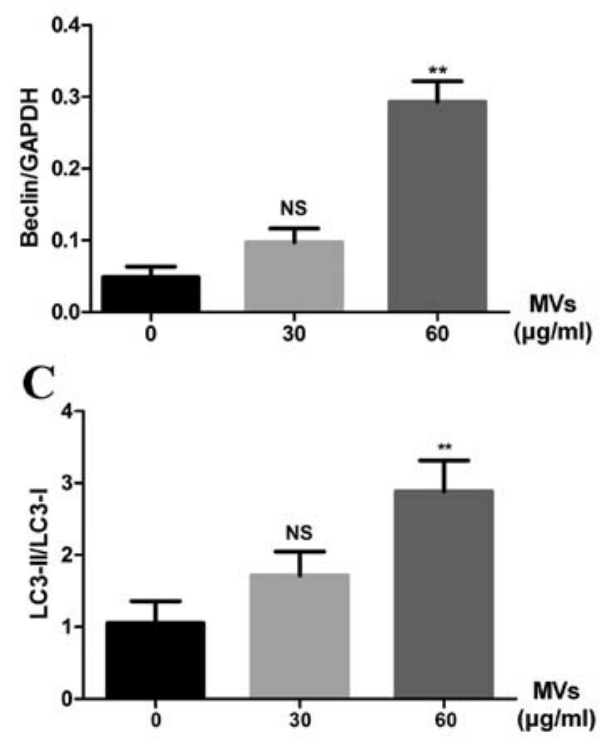

D

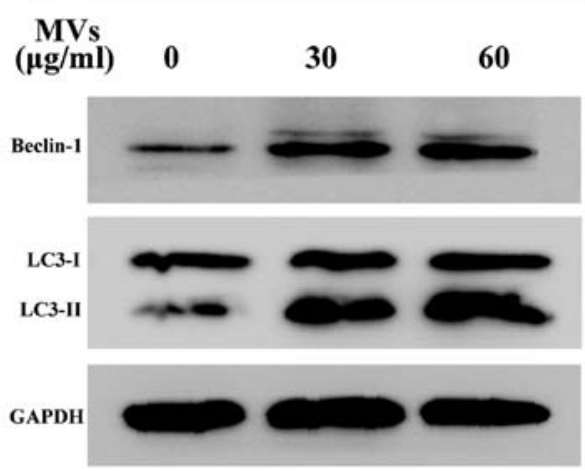

E

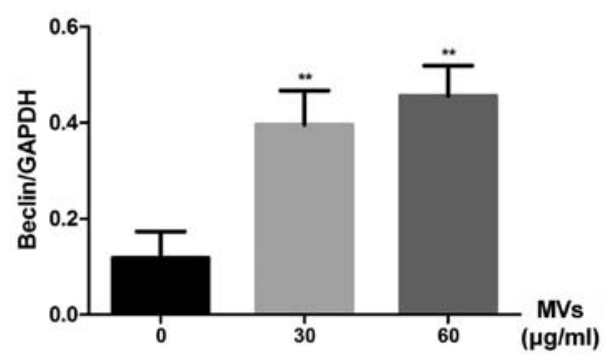

F

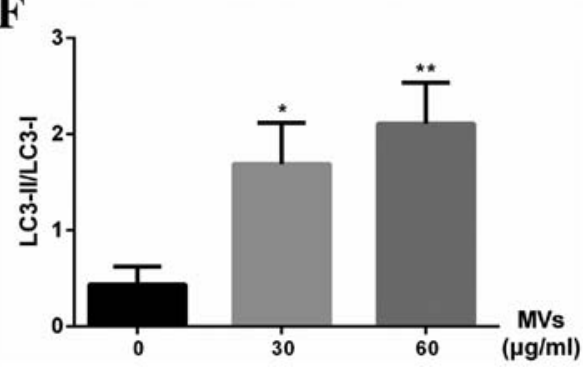

Figure 5. Microvesicles released from human embryonic stem cell derived-mesenchymal stem cells upregulate autophagic activity in K562 and HL60 cells Leukemia cells were incubated with microvesicles for $48 \mathrm{~h}$, and autophagic activity was investigated by western blot analysis (K562) (A) and (HL60) (D). Beclin-1 elevation (K562) (B) and (HL60) (E) and LC3-II conversion (K562) (C) and (HL60) (F) were analyzed using Quantity One v4.62 Software. n=3, ${ }^{*} \mathrm{p}<0.05,{ }^{* *} \mathrm{p}<0.01$. NS, not significant.

exocytose (Fig. 3A). We tested the content of MVs through a BCA protein assay kit resulting in $93.7 \mu \mathrm{g} / 100 \mu \mathrm{l}$.

Considering that paracrine might play an important part in the inhibitory effect, we incubated K562 and HL60 cells with different levels of MVs ranging from 0 to $60 \mu \mathrm{g} / \mathrm{ml}$. Data showed that MVs were able to inhibit the growth of leukemia cells as well as hESC-MSCs. This effect was enhanced by increasing the dose of MVs. However, this inhibitory rate did not increase obviously when the concentration of MVs reached $30 \mu \mathrm{g} / \mathrm{ml}$ (Fig. 3C).

hESC-MSC-MVs upregulate autophagic activity in K562 and HL60 cells. After 48-h incubation with 0 and $30 \mu \mathrm{g} / \mathrm{ml}$ MVs, K562 and HL60 cells were identified under TEM. While treated groups showed many autophagosomes, electron-dense vacuoles, which contained degraded organelles such as mitochondria and endoplasmic reticulum, such vacuoles were absent in control groups (Fig. 4).

To confirm MVs influence autophagic activity in leukemia cells, western blot analysis of Beclin-1 elevation and LC3 conversion was performed. In the experiment groups, K562 and HL60 cells were incubated with 30 and $60 \mu \mathrm{g} / \mathrm{ml}$ of MVs for $48 \mathrm{~h}$. Results confirmed increasing level of Beclin-1 and LC3-II formation, which indicated that MVs could apparently upregulate the autophagic activity compared with untreated groups (Fig. 5A and D). Semiquantitative analysis are shown of Beclin-1 levels (Fig. 5B and E) and LC3 conversion (Fig. 5C and F).

hESC-MSC-MVs promote apoptosis in leukemia cells. K562 and HL60 cells were stained with Annexin V/PI kit and flow cytometric analysis indicated that the apoptosis ratio of leukemia cells increased with the higher concentration of MVs (Fig. 6).

Western blotting was conducted to detect the ratio of Bcl-2 to Bax, which is a common way to assess the level of apoptosis. Results showed that the ratio was significantly decreased in MV-treated groups (Fig. 7).

\section{Discussion}

Previous studies showed that UC-MSCs could inhibit the proliferation of K562 (7) after coculture. BM-MSCs were 


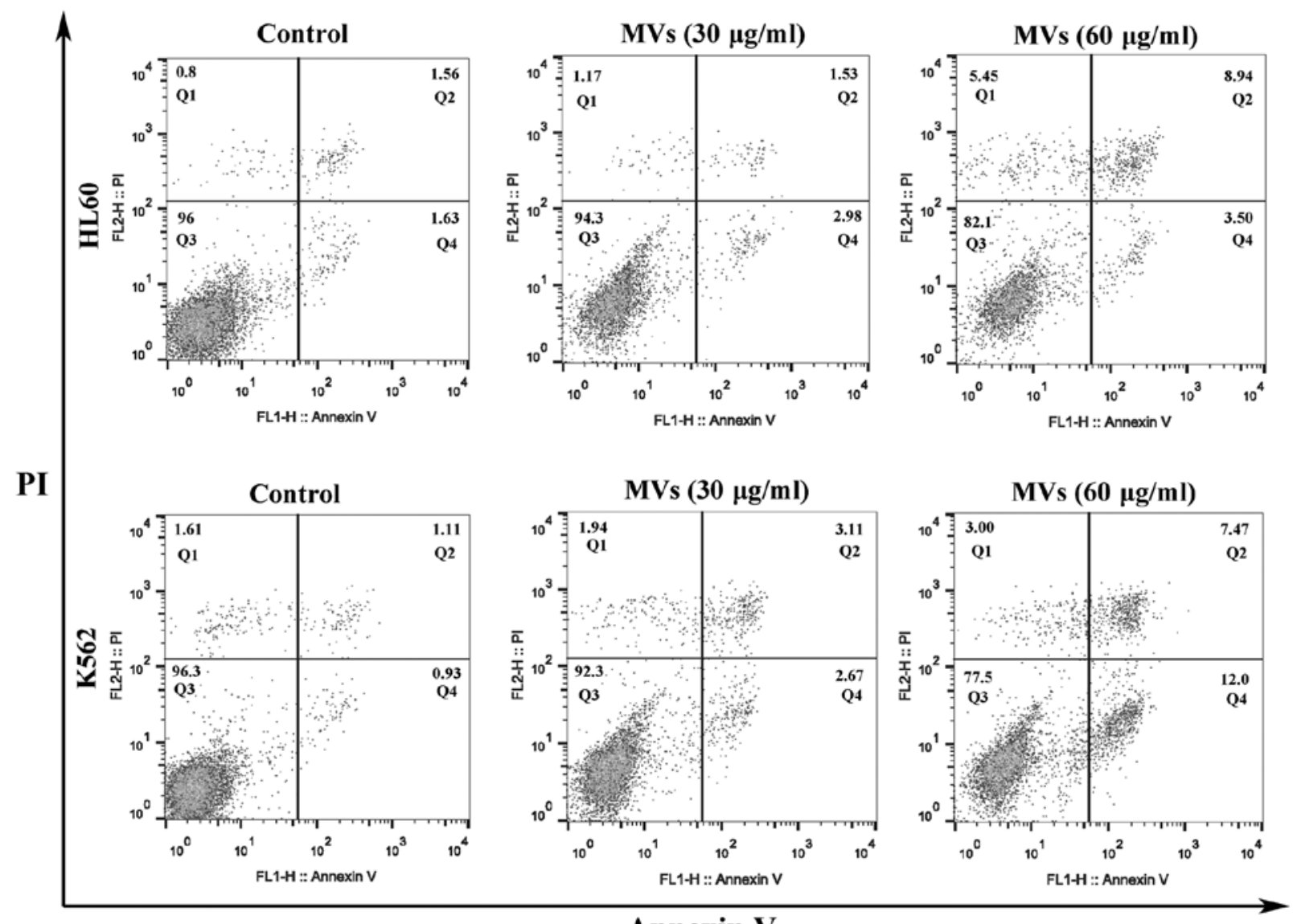

\section{Annexin V}

Figure 6. The apoptosis ratio of K562 and HL60 cells increased with the higher concentration of microvesicles. After 24-h incubation with microvesicles, the proportions of apoptotic cells increased, as detected by FCM assay. One of three representative experiments is shown.

A
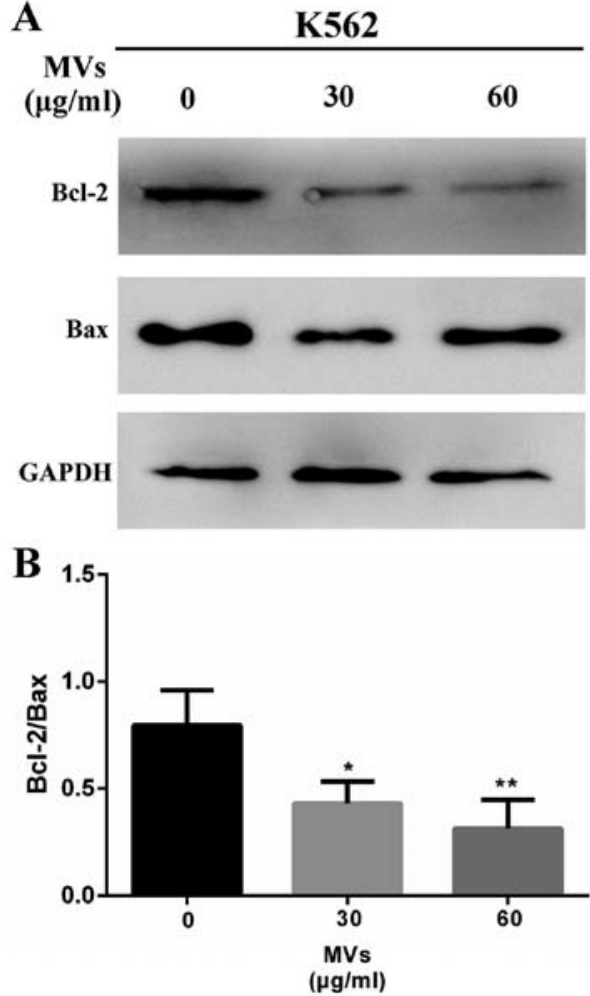

C

MVs

$(\mu \mathrm{g} / \mathrm{ml}) \quad 0 \quad 30$

60

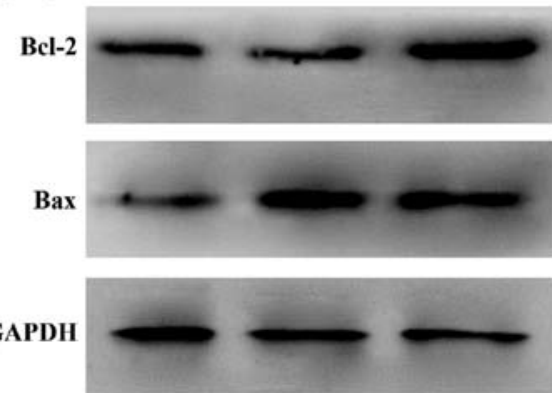

D

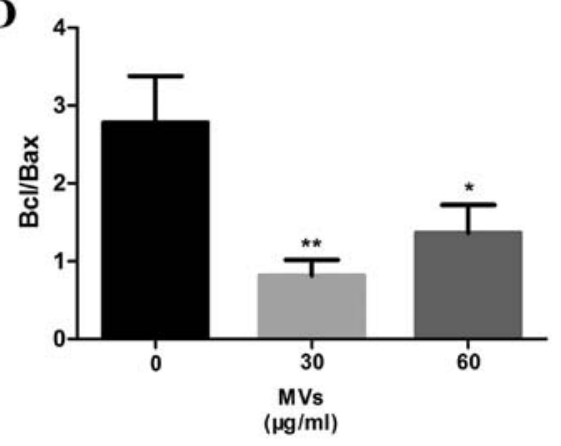

Figure 7. Human embryonic stem cell derived-mesenchymal stem cells reduce the level of Bcl-2/Bax and promote apoptosis. K562 and HL60 cells were incubated with microvesicles for $48 \mathrm{~h}$ in treated groups. Expression levels of Bax and Bcl-2 protein were measured by western blot analysis (K562) (A), (HL60) (C). Additionally, we analyzed the level of Bcl-2/Bax using Quantity One Software (K562) (B), (HL60) (D). $n=3,{ }^{*} \mathrm{p}<0.05,{ }^{* *} \mathrm{p}<0.01$. 
found to inhibit the proliferation of malignant cells of hematopoietic origin $(11,12)$. However, these MSCs had many limitations such as inadequate supply and inevitable invasion when collected. Our previous study focused on the acquisition and characterization of hESC-MSCs and found that they shared many characteristics with traditional MSCs, including morphology, self-renewal and differentiation (8). Particularly, our hESC-MSCs could be cultured for at least 26 passages (data not shown), which might become a better source of MSCs. On the basis of this analysis and previous work, we replaced traditional UC-MSCs or BM-MSCs with hESC-MSCs and received the expected results that they could also inhibit leukemia cells hESC-MSCs. However, it was notable that Ramasamy et al and Goldstein et al reported MSCs could form a cancer stem cell niche in which tumor cells could preserve the potential of proliferating and sustaining the malignant process $(12,13)$. According to the studies of Fonseka et al and Psaila et al the anti-proliferative effect of UC-MSCs contributed to arrest the growth of K562 cells in rest stage (G0/G1 phase), which might confer tumor cells a better survival and self-renewal ability $(7,14)$. The transplantation of human MSCs to mice showed tumorigenicity, attracting attention to this new therapy $(15,16)$. Considering these problems, we focused on the active components in the MSCs culture supernatants.

We observed that the inhibitory effect still existed in Transwell coculture and there was no significant difference compared with the direct contact group, which provided further evidence that paracrine might play an important role. However, Fonseka et al pointed out that the anti-proliferative effect was reduced in Transwell and supernatant groups, and direct cell-to-cell contact might play a significant role (7). This variation might be contributed to different types and pretreatment of MSCs and, more importantly, the culture conditions. In addition, the effect might be not obvious if tumor cells were cocultured with the supernatants of MSCs directly because the concentration of active components within would be too low. Considering the above, we concentrated on the MSC culture supernatants and obtained MVs. Under TEM, MVs were electron-dense vacuoles, several hundred nanometers in diameter and released from the cell membrane surface or by exocytosis $(17,18)$. As expected, MVs inhibited the growth of K562 and HL60 cells in a concentration-dependent manner, which further strengthened the evidence that paracrine or MVs did play an important role. Similar findings were provided by Yang et al. In rheir study, exosomes from ACHN cells inhibited Jurkat T cell proliferation, which also showed a concentrationdependent manner (19). MVs were expected to become new material of biotherapy for cancer. Under electron microscope, we found many electron-dense vacuoles containing extensively degraded organelles such as mitochondria and endoplasmic reticulum in MV-treated groups. These vacuoles were similar to autophagosomes.

Autophagy, or 'self-eating', performs a very important function in many physiological and pathological processes. For example, it provides cells with metabolic precursors as well as energy under starvation and at the same time alleviates stress by removing long lived proteins and damaged organelles (20). However, dysregulated or excessive autophagy certainly can cause autophagic cell death, also called 'the type II programmed cell death' $(21,22)$. In view of this, some anti-neoplastic therapies have focused on inducing autophagy in human cancer cell lines $(23,24)$. TEM was widely perceived as a 'gold standard' test for autophagy, the mark of autophagy under TEM was the presence of autophagosome accumulation. We observed many autophagosomes in MV-treated groups and suspected that MVs might upregulate autophagy in leukemia cells. Then, western blotting was performed to measure the expression level of autophagy-related proteins. In our test, another important finding was that MVs upregulated the Beclin-1 protein level in leukemia cells. Notably, Beclin-1 was the first gene that had been proved to be related with mammalian autophagy (25). It was reported that Beclin-1 could inhibit cancer initiation and showed low levels in human breast carcinoma (26). It should be clearly noted that autophagy is much more than just a promoter or inhibitor of cancers and the relationship between them is intricate and complex (27). Another important problem is the relationship between autophagy and apoptosis. These two programmed cell death pathways were confirmed to coregulate the survival and death of cells $(28,29)$. In some cases, autophagy inhibited apoptosis to adapt to metabolic and environmental stress; in others, it coordinated with apoptosis to result in cell death (30). Cancer cells have various ways to avoid apoptosis, the important means by which organisms deal with defective cells (31). Inducing apoptosis in tumor cells is believed to be a common strategy to fight against cancer (32). Other laboratories have provided evidence that exosomes or MVs could promote apoptosis in tumor cells $(33,34)$. Our data showed that MVs induced autophagy and excessive autophagy might induce apoptosis, which explained in part the inhibitory effect of MVs on leukemia cells. However, the molecular mechanism of leukemia cell autophagy and apoptosis induced by MVs still remains unclear and need further study.

\section{Acknowledgements}

This study was supported by the National Natural Science Foundation of China (grant no. 81571221) and the Natural Science Foundation of Jiangsu Province (grant no. BK20151346).

\section{References}

1. Hu YL, Fu YH, Tabata Y and Gao JQ: Mesenchymal stem cells: A promising targeted-delivery vehicle in cancer gene therapy. J Control Release 147: 154-162, 2010.

2. Drissi H, Gibson JD, Guzzo RM and Xu RH: Derivation and chondrogenic commitment of human embryonic stem cell-derived mesenchymal progenitors. Methods Mol Biol 1340: 65-78, 2015.

3. Brown PT, Squire MW and Li WJ: Characterization and evaluation of mesenchymal stem cells derived from human embryonic stem cells and bone marrow. Cell Tissue Res 358: 149-164, 2014.

4. Zhang H, Bai M, Deng T, Liu R, Wang X, Qu Y, Duan J, Zhang L, Ning T, Ge S, et al: Cell-derived microvesicles mediate the delivery of miR-29a/c to suppress angiogenesis in gastric carcinoma. Cancer Lett 375: 331-339, 2016.

5. Wu S, Ju GQ, Du T, Zhu YJ and Liu GH: Microvesicles derived from human umbilical cord Wharton's jelly mesenchymal stem cells attenuate bladder tumor cell growth in vitro and in vivo. PLoS One 8: e61366, 2013.

6. Fonsato V, Collino F, Herrera MB, Cavallari C, Deregibus MC, Cisterna B, Bruno S, Romagnoli R, Salizzoni M, Tetta C, et al: Human liver stem cell-derived microvesicles inhibit hepatoma growth in SCID mice by delivering antitumor microRNAs. Stem Cells 30: 1985-1998, 2012. 
7. Fonseka M, Ramasamy R, Tan BC and Seow HF: Human umbilical cord blood-derived mesenchymal stem cells (hUCB-MSC) inhibit the proliferation of K562 (human erythromyeloblastoid leukaemic cell line). Cell Biol Int 36: 793-801, 2012.

8. Hu JB, Wang XH, Ma QH, Zhang W, Wang YY, Wen XM. Preliminary investigation on the differentiation of human embryonic stem cells into mesenchymal stem cells. Chin J Clin Lab Sci 31: 276-278, 2013.

9. Gadkari R, Zhao L, Teklemariam T and Hantash BM: Human embryonic stem cell derived-mesenchymal stem cells: An alternative mesenchymal stem cell source for regenerative medicine therapy. Regen Med 9: 453-465, 2014.

10. Bruno S, Collino F, Deregibus MC, Grange C, Tetta C and Camussi G: Microvesicles derived from human bone marrow mesenchymal stem cells inhibit tumor growth. Stem Cells Dev 22: 758-771, 2013.

11. Song N, Gao L, Qiu H, Huang C, Cheng H, Zhou H, Lv S, Chen L and Wang J: Mouse bone marrow-derived mesenchymal stem cells inhibit leukemia/lymphoma cell proliferation in vitro and in a mouse model of allogeneic bone marrow transplant. Int J Mol Med 36: 139-149, 2015.

12. Ramasamy R, Lam EW, Soeiro I, Tisato V, Bonnet D and Dazzi F: Mesenchymal stem cells inhibit proliferation and apoptosis of tumor cells: Impact on in vivo tumor growth. Leukemia 21: 304-310, 2007.

13. Goldstein RH, Reagan MR, Anderson K, Kaplan DL and Rosenblatt M: Human bone marrow-derived MSCs can home to orthotopic breast cancer tumors and promote bone metastasis Cancer Res 70: 10044-10050, 2010.

14. Psaila B, Kaplan RN, Port ER and Lyden D: Priming the 'soil' for breast cancer metastasis: The pre-metastatic niche. Breast Dis 26: 65-74, 2006-2007.

15. Luo F, Liu T, Wang J, Li J, Ma P, Ding H, Feng G, Lin D, Xu Y and Yang K: Bone marrow mesenchymal stem cells participate in prostate carcinogenesis and promote growth of prostate cancer by cell fusion in vivo. Oncotarget 7: 30924-30934, 2016.

16. Burns JS, Abdallah BM, Guldberg P, Rygaard J, Schrøder HD and Kassem M: Tumorigenic heterogeneity in cancer stem cells evolved from long-term cultures of telomerase-immortalized human mesenchymal stem cells. Cancer Res 65: 3126-3135, 2005.

17. Raposo G and Stoorvogel W: Extracellular vesicles: Exosomes, microvesicles, and friends. J Cell Biol 200: 373-383, 2013.

18. Gambim MH, do Carmo AO, Marti L, Veríssimo-Filho S, Lopes LR and Janiszewski M: Platelet-derived exosomes induce endothelial cell apoptosis through peroxynitrite generation: Experimental evidence for a novel mechanism of septic vascular dysfunction. Crit Care 11: R107, 2007.

19. Yang L, Wu X, Wang D, Luo C and Chen L: Renal carcinoma cell-derived exosomes induce human immortalized line of Jurkat T lymphocyte apoptosis in vitro. Urol Int 91: 363-369, 2013.
20. Fulda S and Kögel D: Cell death by autophagy: Emerging molecular mechanisms and implications for cancer therapy. Oncogene 34: 5105-5113, 2015.

21. Kondo Y, Kanzawa T, Sawaya R and Kondo S: The role of autophagy in cancer development and response to therapy. Nat Rev Cancer 5: 726-734, 2005.

22. Yu L, Alva A, Su H, Dutt P, Freundt E, Welsh S, Baehrecke EH and Lenardo MJ: Regulation of an ATG7-beclin 1 program of autophagic cell death by caspase-8. Science 304: 1500-1502, 2004.

23. Borthakur G, Duvvuri S, Ruvolo V, Tripathi DN, Piya S, Burks J, Jacamo R, Kojima K, Ruvolo P, Fueyo-Margareto J, et al: MDM2 inhibitor, nutlin 3a, induces p53 dependent autophagy in acute leukemia by AMP kinase activation. PLoS One 10: e0139254, 2015.

24. Paglin S, Hollister T, Delohery T, Hackett N, McMahill M, Sphicas E, Domingo D and Yahalom J: A novel response of cancer cells to radiation involves autophagy and formation of acidic vesicles. Cancer Res 61: 439-444, 2001.

25. Fu LL, Cheng Y and Liu B: Beclin-1: Autophagic regulator and therapeutic target in cancer. Int J Biochem Cell Biol 45: 921-924, 2013.

26. Liang XH, Jackson S, Seaman M, Brown K, Kempkes B, Hibshoosh $\mathrm{H}$ and Levine B: Induction of autophagy and inhibition of tumorigenesis by beclin 1. Nature 402: 672-676, 1999.

27. White E: The role for autophagy in cancer. J Clin Invest 125 : 42-46, 2015.

28. Yang C, Kaushal V, Shah SV and Kaushal GP: Autophagy is associated with apoptosis in cisplatin injury to renal tubular epithelial cells. Am J Physiol Renal Physiol 294: F777-F787, 2008.

29. Kroemer G and Levine B: Autophagic cell death: The story of a misnomer. Nat Rev Mol Cell Biol 9: 1004-1010, 2008.

30. Gump JM and Thorburn A: Autophagy and apoptosis: What is the connection? Trends Cell Biol 21: 387-392, 2011.

31. Hanahan D and Weinberg RA: Hallmarks of cancer: The next generation. Cell 144: 646-674, 2011.

32. Campisi J: Senescent cells, tumor suppression, and organismal aging: Good citizens, bad neighbors. Cell 120: 513-522, 2005.

33. Zaharie F, Muresan MS, Petrushev B, Berce C, Gafencu GA, Selicean S, Jurj A, Cojocneanu-Petric R, Lisencu CI, Pop LA, et al: Exosome-carried microRNA-375 inhibits cell progression and dissemination via Bcl-2 blocking in colon cancer. J Gastrointestin Liver Dis 24: 435-443, 2015.

34. Ristorcelli E, Beraud E, Mathieu S, Lombardo D and Verine A: Essential role of Notch signaling in apoptosis of human pancreatic tumoral cells mediated by exosomal nanoparticles. Int $\mathrm{J}$ Cancer 125: 1016-1026, 2009. 